\title{
Cognitive Neuroscience in Information Systems Research
}

Behavior research is a major stream in information systems (IS) research. Traditionally, researchers follow theories and paradigms developed in psychology, sociology, and verify their applicability in information technology adoption and related issues. Questionnaire is the major instrument for investigation. This, however, has a limitation in that it is hard to verify whether the reported data truly reflect the actual mental situation of the subject. It is well-known that human subjects have cognitive restrictions in reporting their implicit attitudes. Therefore, a few techniques have been developed to improve data quality and to avoid problems such as common method biases, unreliable or invalid data.

As the advancement of measurement technologies, an emerging trend in cognitive psychology is to use neuroscience knowledge as a foundation to collect evidence for interpreting human behaviors. This new trend also opens a new window for behavior research in information systems because some previously unobservable evidences can now be used to explore insight of IS design and theories. Major journals such as MIS Quarterly, Information Systems Research and Journal of MIS have indicated their strong interests in this new research methodology. Applying cognitive neuroscience methods to IS research is no panacea, however. It must be used cautiously. In this short article, I would like to briefly introduce the neuroscience methodology and its pros and cons.

\section{What is cognitive neuroscience research?}

Cognitive neuroscience is a branch of Psychology and Neuroscience. As described in Wikipedia:

"Cognitive neuroscience is an academic field concerned with the scientific study of biological substrates underlying cognition, with a specific focus on the neural substrates of mental processes. It addresses the questions of how psychological/ cognitive functions are produced by the brain."

A few topics are popular in cognitive neuroscience research: attention, consciousness, learning, memory, risk handling, decision making, emotion, language, and so on. In fact, it is not hard to find linkages between these research areas and IS. For instance, website design can naturally benefit from findings in attention research in neural science. This is why eye-trackers become a popular instrument in user interface design. Functional brain imaging has been applied to study the difference between trust and distrust (Dimoka, 2010).

Behavior economics is probably the first area to adopt neuroscience methods for interpreting decision-making behavior. In a seminal article in neuro-economics, Camerer, et al. (2005) identified six categories of neuroscience methods: brain imaging, single neuron measurement, electrical brain stimulation, psychopathology and brain damage in humans, psychophysical measurement, and diffusion tensor imaging. Among them, brain imaging and psychophysical measurements are more popular in social science research.

(1) Brain imaging: Its major purpose is to identify which area of the brain is activated when a particular task is performed by the subject and interpret the association between brain activation and the stimulus. Three major instruments are used for brain imaging:

- Electro-encephalogram (or EEG) uses electrodes attached to the scalp to measure electrical activity (brain waves) synchronized to stimulus events or behavioral responses (known as Event Related Potentials or ERPs); 
- Positron emission topography (PET) measures blood flow in the brain as a proxy for neural activity;

- Functional magnetic resonance imaging (fMRI) tracks blood flow in the brain using changes in magnetic properties due to blood oxygenation (the "BOLD signal"). This is a new and popular method for the time being.

(2) Psychophysical measurement: Measurement of psychophysiological indicators like heart rate, blood pressure, galvanic skin response (GSR, sweating in the palms), and pupil dilation (pupils dilate in response to arousal) to detect a subject's reaction to a stimulus.

\section{How can neuroscience help IS research?}

Neuroscience research in information systems, which is called neuro IS, catches attention only in recent five years. As defined in Reidl, et al. (2010), Neuro IS is:

"a subfield in the IS literature that relies on neuroscience and neurophysiological theories and tools to better understand the development, use, and impact of information technologies (IT)."

What makes neuro IS research different from other IS research paradigms is that the former has its methodology rooted in cognitive neuroscience methods. It relies on data collected from neuro-scientific instruments and theories developed in neuroscience to interpret the findings. As neuroscience method can provide evidence that is not available in traditional behavior or design science research, it can contribute to information systems research in several ways:

(1) Falsifying/enhancing existing theories. New evidence can be used to examine existing theories that may be falsified or enhanced.

(2) Developing new theories. Adapting neurophysiological knowledge to IS research has the potential of developing new theories for interpreting IS design and/or usage behavior.

(3) Facilitating system design. Deeper knowledge of IS usage may lead to better design of future information systems. For example, knowing how a user views a website would lead to a better webpage design.

(4) Evaluating system design. Neurophysiological evidence may be used to evaluate the design of a system. For instance, the attractiveness of a user interface design may be assessed by the psychophysiological evidence of the experiment subject, instead of a survey questionnaire.

(5) Better measurement of existing constructs. Certain latent constructs measured by reflective indicators may be better assessed with neuroscience methods.

\section{Potential problems in neuroscience research?}

Neuroscience methods are not without limitations, when we apply them to IS research. The following potential problems exist.

(1) Need special training and careful research design. Most IS researchers are not trained to conduct neuroscience studies. Different instruments have their respective strengths and restrictions. For instance, EEG is good in identifying the temporal response of a brain, but is weak in identifying the exact location of the activated brain area, while fMRI is good in identifying the activated spot.

Research design is another issue. Most neuroscience research uses within-subject design, while traditional IS research often adopts between-subject design. The experiment also needs to be simplified in order to find associations between brain activities and actual behavior. In general, experiments beyond two factors are too complicated. It is also 
necessary to have neurocognitive hypothesis in addition to behavior hypothesis in neuro IS research models.

(2) Limited interpretability. As neuroscience is still a developing area, a precise interpretation is sometimes difficult. The exact association between brain activities and behavior may be difficult to prove. It is also hard to conclude causal relationships that most IS researchers would like to see. An analogy of interpreting neuroscience findings is to "infer which system function is working (emotion or cognition) by examining which hard disk area is activated (detected by brain imaging or brain waves)." Currently, we can only use the evidence to tell that an emotion has been activated, but may not be able to tell precisely which emotion it is. For instance, the activation of anterior insular is believed to "process a person's sense of disgust. In social experience it is involved in the processing of norm violations, emotional processing, empathy, and orgasms" (Wikipedia). This kind of interpretational ambiguity (i.e., multiple interpretations of the same observation) may limit the validity of the finding.

To summarize, neuroscience methods provide opportunities to examine design and behavior research in information systems. Although the methods are not without constraints, they do allow us to examine existing issues from a different angle and explore new issues that might not be possible otherwise.

\section{Papers in This Issue}

Two papers are included in this issue. The first one by Huang, Farn, and Jeng reports findings of user behavior in virtual communities. In particular, they divide users into information browsers, information consumers, and information providers. Their motivations and respective behaviors in virtual communities are examined.

The second paper by Lee, Song, and Yoo investigates factors influencing consumers' adoption of Internet protocol TV (IPTV). An online survey was conducted to collect data for hypothesis testing. The result indicates that relative advantage, compatibility, trialability, content diversity, monetary value, personal innovativeness, and social influence have significant direct effects on the consumer's adoption of IPTV. Some moderating effects are also observed.

\section{New Associate Editor}

I would like to welcome a new associate editor, Probir Kumar Banerjee of Swinburn University of Technology in Malaysia. Professor Banerjee's primary research interests include e-business and managerial aspects of information systems.

\section{Ting-Peng Liang}

Editor in Chief

\section{References}

1. Camerer, C., Loewenstein, G. and Prelec, D. (2005), "Neuroeconomics: How Neuroscience Can Inform Economics," Journal of Economic Literature, XLIII, March, pp. 9-64.

2. Dimoka, A. (2010), "What Does the Brain Tell us about Trust and Distrust? Evidence from a Functional Neuroimaging Study," MIS Quarterly, 34(2). (forthcoming)

3. Riedl, R.; Banker, R. D.; Benbasat, I.; Davis, F. D.; Dennis, A. R.; Dimoka, A.; Gefen, D.; Gupta, A.; Ischebeck, A.; Kenning, P.; Müller-Putz, G.; Pavlou, P. A.; Straub, D. W.; vom Brocke, Jan; and Weber, B. (2010), "On the Foundations of NeurolS: Reflections on the Gmunden Retreat 2009," Communications of the Association for Information Systems, 27(15). 